\title{
A note on the Cauchy problem for the 2D generalized Zakharov-Kuznetsov equations
}

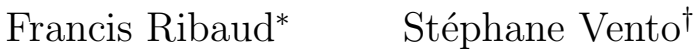

\begin{abstract}
In this note we study the generalized 2D Zakharov-Kuznetsov equations $\partial_{t} u+\Delta \partial_{x} u+u^{k} \partial_{x} u=0$ for $k \geq 2$. By an iterative method we prove the local well-posedness of these equations in the Sobolev spaces $H^{s}\left(\mathbb{R}^{2}\right)$ for $s>1 / 4$ if $k=2, s>5 / 12$ if $k=3$ and $s>1-2 / k$ if $k \geq 4$.
\end{abstract}

Keywords: KdV-like equations, Cauchy problem

AMS Classification: 35Q53, 35B65, 35Q60

\section{Introduction}

In this short note, we are interested with the Cauchy problem associated to the generalized Zakharov-Kuznetsov (gZK) equations

$$
u_{t}+\Delta u_{x}+u^{k} u_{x}=0,
$$

in two-dimensional space and for $k=2,3,4, \ldots$. These equations are natural multi-dimensional generalizations of the well-known generalized Kortewegde Vries equations and have been derived in [8] when $k=1$ to model the propagation of nonlinear ionic-sonic waves in a magnetized plasma.

We give sharp results concerning the well-posedness issue in standard Sobolev spaces $H^{s}\left(\mathbb{R}^{2}\right)$ for suitable $s \in \mathbb{R}$. This work follows and use similar technics as in the paper [6] where we proved that the 3D associated problem for $k=1$ is locally well-posed in $H^{1^{+}}\left(\mathbb{R}^{3}\right)$.

Remark that the Sobolev spaces $\dot{H}^{s}\left(\mathbb{R}^{2}\right)$ are invariant by the natural rescaling of the equation as soon as $s=s_{k}:=1-2 / k$. Thus a natural question is whether (gZK) is well-posed in $H^{s}\left(\mathbb{R}^{2}\right)$ for $s>s_{k}$.

\footnotetext{
${ }^{*}$ Laboratoire d'Analyse et de Mathématiques Appliquées - Université Paris-Est - 5 Bd. Descartes - Champs-Sur-Marne, 77454 Marne-La-Vallée Cedex 2 (FRANCE)

${ }^{\dagger}$ Laboratoire Analyse, Géométrie et Applications - Université Paris 13 - Institut Galilée, 99 avenue J.B. Clément,93430 Villetaneuse (FRANCE)
} 
Theorem 1.1. For any $u_{0} \in H^{s}\left(\mathbb{R}^{2}\right)$ with

$$
\begin{cases}s>1 / 4 & \text { if } k=2 \\ s>5 / 12 & \text { if } k=3 \\ s>1-2 / k & \text { if } k \geq 4\end{cases}
$$

there exist $T>0$, a Banach space $X_{T}^{s}$ and a unique solution $u$ of the Cauchy problem associated to (1.1) with $u(0)=u_{0}$ such that $u \in X_{T}^{s} \cap$ $C_{b}\left([0, T], H^{s}\left(\mathbb{R}^{2}\right)\right)$. Moreover, the flow-map $u_{0} \mapsto u$ is Lipschitz on every bounded set of $H^{s}\left(\mathbb{R}^{2}\right)$.

This theorem improves the recent works of Farah, Linares and Pastor in [2]-[4]-[5] where local well-posedness was obtained in $H^{s}\left(\mathbb{R}^{2}\right)$ for $s>3 / 4$ if $2 \leq k \leq 8$ and $s>s_{k}$ if $k>8$.

In view of the ill-posedness result obtained in [5], Theorem[1.1] is optimal (up to the end point) for $k \geq 4$ whereas in the particular cases $k=2$ and 3 , we still have a gap (respectively $1 / 4$ and $1 / 12$ ) compared with the scaling index. Concerning the end point $s=s_{k}$, local well-posedness could perhaps be reached by following the strategy developed in [7], but with a flow-map only continuous.

In a standard way our proof is based on a fixed point scheme applied to the Duhamel formulation of (1.1):

$$
u(t)=U(t) u_{0}-\frac{1}{k+1} \int_{0}^{t} U\left(t-t^{\prime}\right) \partial_{x}\left(u^{k+1}\right)\left(t^{\prime}\right) d t^{\prime} .
$$

where $U(t)=e^{-t \Delta \partial_{x}}$ denotes the propagator associated with the linear part of (1.1). Following the works of Kenig, Ponce and Vega [3] on the $\mathrm{KdV}$ equation, we use in a crucial way some sharp dispersive estimates for free solutions. More precisely, these estimates are the well known Kato smoothing effect

$$
\|\nabla U(t) \varphi\|_{L_{x}^{\infty} L_{y T}^{2}} \lesssim\|\varphi\|_{L^{2}}
$$

which allows to gain one derivative in each spatial direction, and the maximal in time estimate

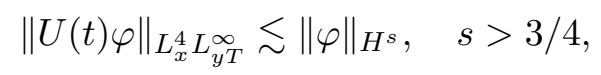

proved in [4]. On the other hand, similarly to the generalized KdV equations, the previous bound is no more sufficient to deal with low non-linearities $(k=2,3)$ and we need the following $L_{x}^{2}$ based maximal in time estimate (see [1])

$$
\|U(t) \varphi\|_{L_{x}^{2} L_{y T}^{\infty}} \lesssim\|\varphi\|_{H^{s}}, \quad s>3 / 4
$$




\section{Proof of the main result}

\subsection{The case $k \geq 4$}

As mentioned in the introduction, we want to take advantage of the $L_{x}^{4} L_{y T}^{\infty}$ linear estimate (1.4). This motivates the choice of our resolution space:

$$
X_{T}^{s}=\left\{u \in C_{b}\left([0, T], H^{s}\left(\mathbb{R}^{2}\right)\right):\|u\|_{X_{T}^{s}}<\infty\right\},
$$

where

$$
\|u\|_{X_{T}^{s}}=\|u\|_{L_{T}^{\infty} H_{x y}^{s}}+\left\|\langle\nabla\rangle^{s+1} u\right\|_{L_{x}^{\infty} L_{y T}^{2}}+\left\|\langle\nabla\rangle^{s-3 / 4^{+}} u\right\|_{L_{x}^{4} L_{y T}^{\infty}} .
$$

Combining estimates (1.3)-(1.4) as well as the straightforward bound

$$
\|U(t) \varphi\|_{L_{T}^{\infty} L^{2}} \lesssim\|\varphi\|_{L^{2}}
$$

we get

$$
\|U(t) \varphi\|_{X_{T}^{s}} \lesssim\|\varphi\|_{H^{s}} .
$$

Note that the bound for the second term can be handled by using a low-high frequencies decomposition and next Bernstein inequality and estimate (1.3). Having the linear part under control, it remains to deal with the integral term. It is not too hard to adapt the proofs of Propositions 3.5-3.6-3.7 in [6] to deduce

$$
\left\|\int_{0}^{t} U\left(t-t^{\prime}\right) \partial_{x} u^{k+1}\left(t^{\prime}\right) d t^{\prime}\right\|_{X_{T}^{s}} \lesssim\left\|\langle\nabla\rangle^{s-1} \partial_{x} u^{k+1}\right\|_{L_{x}^{1} L_{y T}^{2}} .
$$

The multi-dimensional version of Theorem A.13 in [3] applies and leads to

$$
\begin{aligned}
\left\|\langle\nabla\rangle^{s-1} \partial_{x} u^{k+1}\right\|_{L_{x}^{1} L_{y T}^{2}} & \lesssim\left\|\langle\nabla\rangle^{s} u^{k+1}\right\|_{L_{x}^{1} L_{y T}^{2}} \\
& \lesssim\left\|\langle\nabla\rangle^{s} u\right\|_{L_{x}^{7+} L_{y T}^{14 / 3^{-}}}\|u\|_{L_{x}^{7 k / 6^{-}} L_{y T}^{7 k / 2+^{+}}}^{k} .
\end{aligned}
$$

We claim that the first product in the right hand side of (2.4) is controlled by the $X_{T}^{s}$ norm of $u$. Indeed, an interpolation argument shows that

$$
\left\|\langle\nabla\rangle^{\alpha} u\right\|_{L_{x}^{p} L_{y T}^{q}} \lesssim\|u\|_{X_{T}^{s}}
$$

as soon as there exists $\theta \in[0,1]$ such that

$$
\frac{1}{p}=\frac{1-\theta}{4}, \quad \frac{1}{q}=\frac{\theta}{2}, \quad \alpha=\left(s+\frac{7 \theta-3}{4}\right)^{-} .
$$


Taking $\alpha=s$, i.e. $\theta=3 / 7^{+}$, it follows that

$$
\left\|\langle\nabla\rangle^{s} u\right\|_{L_{x}^{7^{+}} L_{y T}^{14 / 3^{-}}} \lesssim\|u\|_{X_{T}^{s}}
$$

If we choose now $\theta=4 / 7 k$ in (2.5), we infer

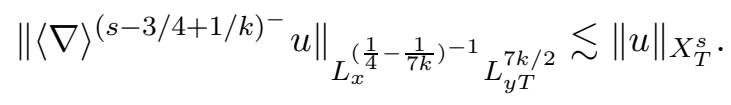

In order to get the desired contraction factor, we will interpolate this inequality with the bound

$$
\left\|\langle\nabla\rangle^{(s-1)^{+}} u\right\|_{L_{x y T}^{\infty-}} \lesssim T^{0^{+}}\left\|\langle\nabla\rangle^{s} u\right\|_{L_{T}^{\infty} L_{x y}^{2}} \lesssim T^{0^{+}}\|u\|_{X_{T}^{s}} .
$$

This leads to

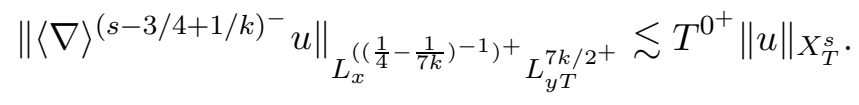

By virtue of the Sobolev inequalities, we get

$$
\begin{aligned}
& \|u\|_{L_{x}^{7 k / 6^{-}} L_{y T}^{7 k / 2^{+}}} \lesssim\left\|\langle\nabla\rangle^{(1 / 4-1 / k)^{+}} u\right\|_{L_{x}^{\left(\left(\frac{1}{4}-\frac{1}{7 k}\right)^{-1}\right)^{+}}}{L_{y T}^{7 k / 2^{+}}}
\end{aligned}
$$

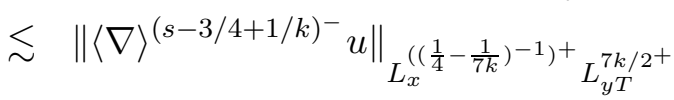

$$
\begin{aligned}
& \lesssim T^{0^{+}}\|u\|_{X_{T}^{s}}
\end{aligned}
$$

for $s-3 / 4+1 / k>1 / 4-1 / k$, that is $s>s_{k}$. Gathering together (2.2)(2.3) $-(2.4)-(2.7)$ and (2.8) we infer that

$$
\|F(u)\|_{X_{T}^{s}} \lesssim\left\|u_{0}\right\|_{H^{s}}+T^{0^{+}}\|u\|_{X_{T}^{s}}^{k+1},
$$

where $F(u)$ denote the right hand side of (1.2). The well-posedness result follows then from standard arguments.

\subsection{The case $k=2$}

The proof in this case follows the same lines as in the case $k \geq 4$, but with the $L_{x}^{4}$ norm replaced with a $L_{x}^{2}$ maximal in time norm. So let us endow the $X_{T}^{s}$ space with the norm

$$
\|u\|_{X_{T}^{s}}=\|u\|_{L_{T}^{\infty} H_{x y}^{s}}+\left\|\langle\nabla\rangle^{s+1} u\right\|_{L_{x}^{\infty} L_{y T}^{2}}+\left\|\langle\nabla\rangle^{s-3 / 4^{+}} u\right\|_{L_{x}^{2} L_{y T}^{\infty}},
$$


for any $s>1 / 4$. Using now (1.5), we easily see that

$$
\left\|U(t) u_{0}\right\|_{X_{T}^{s}} \lesssim\left\|u_{0}\right\|_{H^{s}}
$$

and

$$
\left\|\int_{0}^{t} U\left(t-t^{\prime}\right) \partial_{x} u^{3}\left(t^{\prime}\right) d t^{\prime}\right\|_{X_{T}^{s}} \lesssim\left\|\langle\nabla\rangle^{s-1} \partial_{x} u^{3}\right\|_{L_{x}^{1} L_{y T}^{2}} .
$$

Again, the fractional Leibniz rule yields the bound

$$
\left\|\langle\nabla\rangle^{s-1} \partial_{x} u^{3}\right\|_{L_{x}^{1} L_{y T}^{2}} \lesssim\left\|\langle\nabla\rangle^{s} u\right\|_{L_{x}^{7 / 2^{+}} L_{y T}^{14 / 3^{-}}}\|u\|_{L_{x}^{14 / 5^{-}} L_{y T}^{7^{+}}}^{2}
$$

By interpolation, we get

$$
\left\|\langle\nabla\rangle^{\alpha} u\right\|_{L_{x}^{p} L_{y T}^{q}} \lesssim\|u\|_{X_{T}^{s}}
$$

for $\alpha, p$ and $q$ satisfying

$$
\frac{1}{p}=\frac{1-\theta}{2}, \quad \frac{1}{q}=\frac{\theta}{2}, \quad \alpha=\left(s+\frac{7 \theta-3}{4}\right)^{-}
$$

for $0 \leq \theta \leq 1$. On one hand, we deduce that

$$
\left\|\langle\nabla\rangle^{s-1} \partial_{x} u^{3}\right\|_{L_{x}^{1} L_{y T}^{2}} \lesssim\|u\|_{X_{T}^{s}}
$$

were we took $\theta=3 / 7^{+}$in (2.11). On the other hand, for $\theta=2 / 7^{-}$, we infer

$$
\left\|\langle\nabla\rangle^{(s-1 / 4)^{-}} u\right\|_{L_{x}^{14 / 5^{-}} L_{L_{T}}^{7^{+}}} \lesssim\|u\|_{X_{T}^{s}},
$$

which interpolated with (2.6) gives

$$
\left\|\langle\nabla\rangle^{(s-1 / 4)^{-}} u\right\|_{L_{x}^{14 / 5^{-}} L_{y T}^{7+}} \lesssim T^{0^{+}}\|u\|_{X_{T}^{s}}
$$

This yields the desired result for $s>1 / 4$.

\subsection{The case $k=3$}

Now we consider the intermediate case $k=3$. To prove our result, we define the resolution space as the intersection of the two previous spaces, i.e. equipped with the norm

$$
\|u\|_{X_{T}^{s}}=\|u\|_{L_{T}^{\infty} H_{x y}^{s}}+\left\|\langle\nabla\rangle^{s+1} u\right\|_{L_{x}^{\infty} L_{y T}^{2}}+\left\|\langle\nabla\rangle^{s-3 / 4^{+}} u\right\|_{\left(L_{x}^{2} \cap L_{x}^{4}\right) L_{y T}^{\infty}} .
$$


Actually we don't require the full range $L_{x}^{2} \cap L_{x}^{4}$ for the maximal in time norm, but only

$$
\left\|\langle\nabla\rangle^{s-3 / 4^{+}} u\right\|_{L_{x}^{3} L_{y T}^{\infty}} \lesssim\|u\|_{X_{T}^{s}} .
$$

According to (2.2) $-(2.3)-(2.9)-(2.10)$ we have

$$
\|F(u)\|_{X_{T}^{s}} \lesssim\left\|u_{0}\right\|_{H^{s}}+\left\|\langle\nabla\rangle^{s} u^{4}\right\|_{L_{x}^{1} L_{y T}^{2}}
$$

Using again the Leibniz rule for fractional derivatives, we infer

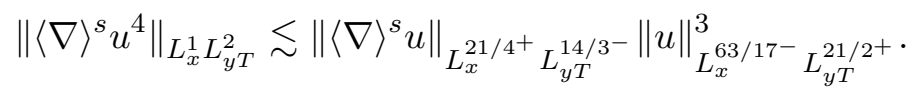

From an interpolation argument with (2.12), we easily check that both these norms are acceptable as soon as $s>5 / 12$.

\section{References}

[1] A. V. Faminskii, The Cauchy problem for the Zakharov-Kuznetsov equation. Differential Equations 31 (1995), no. 6, 1002-1012

[2] L.G. Farah, F. Linares and A. Pastor, A note on the 2D generalized Zakharov-Kuznetsov equation: local, global, and scattering results. 2011, arXiv:1108.3714

[3] C E. Kenig, G. Ponce and L. Vega, Well-posedness and scattering results for the generalized Korteweg-de Vries equation via the contraction principle. Comm. Pure Appl. Math. 46 (1993), no. 4, 527-620.

[4] F. Linares and A. Pastor, Well-posedness for the two-dimensional modified Zakharov-Kuznetsov equation. SIAM J. Math. Anal. 41 (2009), no. 4, 1323-1339.

[5] F. Linares and A. Pastor, Local and global well-posedness for the $2 \mathrm{D}$ generalized Zakharov-Kuznetsov equation. J. Funct. Anal. 260 (2011), no. 4, 1060-1085.

[6] F. Ribaud and S. Vento, Well-posedness results for the 3D ZakharovKuznetsov equation. Preprint, arXiv:1111.2850

[7] S. Vento, Well-posedness for the generalized Benjamin-Ono equations with arbitrary large initial data in the critical space. Int. Math. Res. Not. IMRN 2010, no. 2, 297-319.

[8] V.E. Zakharov and E.A. Kuznetsov, On three dimensional solitons. Sov. Phys. JETP., 39 (1974), 285-286. 